To appear in ApJ December 20, 2007, v671n2 issue.

Preprint typeset using LATEX style emulateapj v. 08/22/09

\title{
CANGAROO-III SEARCH FOR GAMMA RAYS FROM SN 1987A AND THE SURROUNDING FIELD
}

\author{
R. Enomoto ${ }^{1}$ G. V. Bicknell ${ }^{2}$ R. W. Clay ${ }^{3}$ P. G. Edwards $^{4}$ S. Gunji ${ }^{5}$ S. Hara ${ }^{6}$ T. Hattori ${ }^{7}$ S. Hayashi ${ }^{8}$

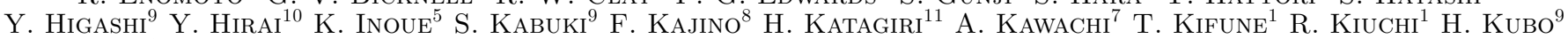 \\ J. Kushida ${ }^{7}$ T. Mizukami ${ }^{9}$ R. Mizuniwa ${ }^{7}$ M. Mori ${ }^{1}$ H. Muraishi $^{12}$ T. NAITo ${ }^{13}$ T. NakAmori ${ }^{9}$ S. NaKano ${ }^{9}$ D. Nishida ${ }^{9}$ \\ K. Nishijima ${ }^{7}$ M. Ohishi ${ }^{1}$ Y. Sakamoto ${ }^{7}$ A. Seki ${ }^{7}$ V. Stamatescu ${ }^{3}$ T. Suzuki ${ }^{10}$ D. L. Swaby ${ }^{3}$ T. Tanimori ${ }^{9}$

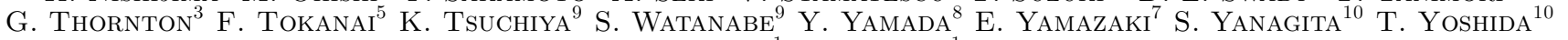 \\ T. YOSHIKOSHI ${ }^{1}$ Y. YUKAWA ${ }^{1}$ \\ To appear in ApJ December 20, 2007, v671n2 issue.
}

\begin{abstract}
Optical images of SN 1987A show a triple ring structure. The inner (dust) ring has recently increased in brightness and in the number of hot spots suggesting that the supernova shock wave has collided with the dense pre-existing circumstellar medium, a scenario supported by radio and X-ray observations. Such a shocked environment is widely expected to result in the acceleration of charged particles, and the accompanying emission of very high energy gamma-rays. Here, we report the results of observations made in 2004 and 2006 which yield upper limits on the $\mathrm{TeV}$ gamma-ray flux, which are compared with a theoretical prediction. In addition, we set upper limits on the TeV flux for four high energy objects which are located within the same field of view of the observation: the superbubble 30 Dor C, the Crab-like pulsar PSR B0540-69, the X-ray binary LMC X-1, and the supernova remnant N157B.
\end{abstract}

Subject headings: gamma rays: search — supernova: individual (SN 1987A)

\section{INTRODUCTION}

SN1987A was the closest observed supernova in 380 years, and the evolution of the remnant of this supernova has been studied in great detail over the past twenty years. The radio intensity is growing and its rate of increase is increasing, with the spectral index being observed to flatten (Stavelev-Smith et al. 2007). Imaging at radio wavelengths has enabled the expansion of the inner ring to be traced, and is revealing increasing structure in the inner ring. The X-ray fluxes observed with $\mathrm{XMM}$ and Chandra also continue to rise almost exponentially (Habert et al. 2006; Park et al. 2007). Around $\sim 4000$ days after the supernova the $\mathrm{X}$-ray light curve increased dramatically, attributed to the arrival of the supernova blast wave at the equatorial ring of circum-

1 Institute for Cosmic Ray Research, University of Tokyo, Kashiwa, Chiba 277-8582, Japan

2 Research School of Astronomy and Astrophysics, Australian National University, ACT 2611, Australia

3 School of Chemistry and Physics, University of Adelaide, SA 5005, Australia

4 Paul Wild Observatory, CSIRO Australia Telescope National Facility, CSIRO, Narrabri, NSW 2390, Australia

5 Department of Physics, Yamagata University, Yamagata, Yamagata 990-8560, Japan

6 Ibaraki Prefectural University of Health Sciences, Ami, Ibaraki 300-0394, Japan

7 Department of Physics, Tokai University, Hiratsuka, Kanagawa 259-1292, Japan

8 Department of Physics, Konan University, Kobe, Hyogo 6588501, Japan

9 Department of Physics, Kyoto University, Sakyo-ku, Kyoto 606-8502, Japan

10 Faculty of Science, Ibaraki University, Mito, Ibaraki 310-8512, Japan

11 Department of Physical Science, Hiroshima University, Higashi-Hiroshima, Hiroshima 739-8526, Japan

12 School of Allied Health Sciences, Kitasato University, Sagamihara, Kanagawa 228-8555, Japan

13 Faculty of Management Information, Yamanashi Gakuin University, Kofu, Yamanashi 400-8575, Japan stellar material. There is some evidence that the X-ray flux is mainly thermal, indicating the blast-wave interaction with dense matter (Park et al. 2007).

Berezhko \& Ksenofontov (2006) compared radio and X-ray data with their calculations and concluded that there is a high (amplified) magnetic field inside the supernova remnant. The predicted $\mathrm{TeV}$ gamma-ray flux, resulting mostly from the decay of neutral pions produced in interactions of the accelerated cosmic rays, is almost one order below the level obtained in previous searches by Imaging Atmospheric Cherenkov Telescopes (IACTs) such as CANGAROO-II in 2001 (Enomoto et al. 2003) and H.E.S.S. in 2003 (Rowell et al. 2005). The wide field of view searched by H.E.S.S. did, however, yield marginal excesses in a region north-east of SN 1987A and at LMC $\mathrm{X}-1$.

CANGAROO-III is one of two stereoscopic IACTs located in the southern hemisphere. With a three-fold coincidence, the sensitivity is significantly improved from the previous single telescope (CANGAROO-II). We also increased the observation period by a factor of two in the 2006 observations compared to the 2004 and 2001 seasons. We here report the results of these observations.

The previous H.E.S.S. analysis considered not only SN 1987A but also four other high energy objects within the same field of view (Rowell et al. 2005). We follow that idea and present upper limits on the integral gamma-ray fluxes from the super-bubble 30 Dor C, the Crab-like pulsar PSR B0540-69, the X-ray binary LMC X-1, and the supernova remnant N157B. Super-bubbles are expected to be efficient accelerators of cosmic rays (Parizot et al. 2004) and a shell feature near the star 30 Dor C, within 5 arcmin of SN 1987A, shows an indication of nonthermal hard X-ray emission (Bamba et al. 2004). The energy required to produce the bubble is estimated to be $7 \times 10^{51} \mathrm{erg}$, which is higher than generally expected for a single supernova remnant Ueno, Bamba, \& Kovama 
2003). The other three sources belong to categories of high-energy astrophysical objects that may accelerate particles to $\mathrm{TeV}$ energies, but, considering their large distance of $50 \mathrm{kpc}$, they are less likely to be detected by current IACTs than their Galactic counterparts. However, as they are well within the field of view of the CANGAROO-III telescopes, and given the marginal H.E.S.S. excess toward LMC X-1, it is straightforward to search for any evidence of gamma-ray emission from them.

\section{CANGAROO-III STEREOSCOPIC SYSTEM}

The CANGAROO-III stereoscopic system consists of four imaging atmospheric Cherenkov telescopes located near Woomera, South Australia $\left(31^{\circ} \mathrm{S}, 137^{\circ} \mathrm{E}\right)$. Each telescope has a $10 \mathrm{~m}$ diameter segmented reflector consisting of 114 spherical mirrors each of $80 \mathrm{~cm}$ diameter (Kawachi et al. 2001), providing a total light collecting area of $57.3 \mathrm{~m}^{2}$. The spherical segments are mounted on a parabolic frame with a focal length of $8 \mathrm{~m}$. The first telescope, T1, which was the CANGAROO-II telescope (Enomoto et al. 2002a), was not used for these observations due to its smaller field of view and higher energy threshold. The second, third, and fourth telescopes (T2, T3, and T4) were used for the observations described here. The camera systems for T2, T3, and T4 are identical and are described in Kabuki et al. (2003). The telescopes are located at the east (T1), west (T2), south (T3) and north (T4) corners of a diamond with sides of $\sim 100 \mathrm{~m}$ (Enomoto et al. 2002b).

\section{OBSERVATIONS}

The observations were carried out in the periods from 2004 Nov 11 to 14 (MJD 53320-53323) and from 2006 Dec 12 to 27 (MJD 54081-54096) using "wobble mode" in which the pointing position of each telescope was shifted in declination between \pm 0.5 degree every 20 minutes (Daum et al. 1997) from the target: (RA, dec $[\mathrm{J} 2000])=\left(83.866139^{\circ},-69.269577^{\circ}\right)$. We took no OFF source runs. The most sensitive region of the field of view is within one degree of the average pointing position. LMC X-1 is located 0.6 degrees south-east from SN 1987A. Considering our angular resolution of 0.24 degree, there is some overlap between SN 1987A, 30 Dor C, PSR B0540-69, and N157B.

In the 2004 observation, data with GPS time stamps were recorded for T2, T3 and T4 individually when more than four photomultiplier (PMT) signals exceeded 7.6 photoelectrons (p.e.). In the offline analysis stage we combined the data when the three telescope's GPS times coincided. In the 2006 observation, a hardware coincidence was used to select any two triggered telescopes (Nishijima et al. 2005). The images in all three telescopes were required to have clusters of at least five adjacent pixels exceeding a 5 p.e. threshold (offline three-fold coincidence). The event rate was reduced to $5 \sim 8 \mathrm{~Hz}$ by this criterion depending on the elevation angle and year. Based on time dependence of these rates we can remove data taken in cloudy conditions. The effective observation times for 2004 and 2006 were 632 and 1316 min, respectively. The corresponding mean zenith angles were $42.1^{\circ}$ and $40.0^{\circ}$.

The light collecting efficiencies, including the reflectivity of the segmented mirrors, the light guides, and the quantum efficiencies of the photomultiplier tubes were monitored by a muon-ring analysis (Enomoto et al. 2006a) with the individual trigger data in the same periods. The light yield per unit arc-length is approximately proportional to the light collecting efficiencies. Deterioration is mostly due to dirt and dust settling on the mirrors.

\section{ANALYSIS}

Here, we briefly describe the analysis procedures, which are identical with those described in Kabuki et al. (2007). More details can be found in Enomoto et al. (2006a) and Enomoto et al. (2006b).

First, the image moments (Hillas 1985) were calculated for the three telescopes. The incident gamma-ray direction was determined by minimizing the sum of squared widths $\left(\chi_{0}^{2}\right.$ : weighted by the photon yield) of the three images seen from the assumed position (fitting parameter) with a constraint on the distances from the intersection point to each image center.

In order to derive the gamma-ray likeliness, we used the Fisher Discriminant (hereafter FD) (Fisher 1936; Enomoto et al. 2006a). Input parameters were

$$
\vec{P}=(W 2, W 3, W 4, L 2, L 3, L 4),
$$

where $\quad W 2, W 3, W 4, L 2, L 3, L 4$ are energy-corrected widths and lengths for the T2, T3, and T4 and assume that a linear combination of

$$
F D=\vec{\alpha} \cdot \vec{P}
$$

provides the best separation between signal and background, then the set of linear coefficients $(\vec{\alpha})$ should be uniquely determined as

$$
\vec{\alpha}=\frac{\vec{\mu}_{s i g}-\vec{\mu}_{B G}}{E_{s i g}+E_{B G}},
$$

where $\vec{\mu}$ is a vector of the mean value of $\vec{P}$ for each sample and $E$ is their correlation matrix. We previously used it in Vela Pulsar analysis (Enomoto et al. 2006a) to separate "sharp" (gamma-ray-like) images from "smeared" (background) ones. The values of $\vec{\mu}_{\text {sig }}, \vec{\mu}_{B G}, E_{\text {sig }}$, and $E_{B G}$ can be calculated from the Monte-Carlo and observational data (OFF-source runs), respectively.

We rejected events with any hits in the outermost layer of a camera ("edge cut"). These rejected events suffer from finite deformations, especially in the length distribution, which results in deformations of the $F D$.

We then derived $F D$ distributions position by position. Comparing those in the signal region and control background region, we can determine the gamma-raylike events. Here, we use Monte-Carlo simulations to determine the $F D$ distribution of gamma-ray signals. Note that in the gamma-ray simulations we used a spectrum proportional to $E^{-2.1}$. The fit of the $F D$ distribution of source position with the above emulated signal and control background functions were carried out, to derive the number of gamma-ray-like events. This is a oneparameter fitting with the constraint that sum of signal and background events corresponds to the total number of events. These coefficients can be derived exactly analytically. 


\section{RESULTS}

The threshold of this analysis is considered to be $\sim 1 \mathrm{TeV}$ which is higher than the typical CANGAROOIII threshold due to the larger zenith angle of the observations, of around 40 degrees. In order to derive morphology, we segmented the field of view into $0.2 \times 0.2$ degree $^{2}$ square bins. The $F D$ distributions for corresponding bins are made and fitted. The control-background region is defined as the second closest layer, which is more than 0.3 degree from the center of target region, i.e., larger than the 0.24 degree point spread function (PSF). The statistics of the control-background is, therefore, sixteen times larger than that of signal bin. The results are shown in Figs. 1. The left panel is obtained from the 2004 data and the right from 2006. The average telescope pointing position is indicated by the cross centered and labeled as "SN 1987A", with the other four high energy objects within this field of view indicated by crosses. Our sensitivity is considered to be limited up to one degree in radius from the center. The PSF is considered to be a 0.24 degree radius circle. The significance distributions (excess divided by the statistical error) are approximately normal (Gaussian) distributions. The best fit Gaussians have mean values of $-0.31 \pm 0.08$ (2004) and $-0.18 \pm 0.09$ (2004), and standard deviations of $1.21 \pm 0.06$ (2004) and $1.31 \pm 0.06$ (2006), within systematic uncertainties.

At first we investigate the region of SN 1987A. Note that this region at some energies is contaminated by 30 Dor $\mathrm{C}$, a highly energetic source. To indicate the excess distribution from its center, we show so-called $\theta^{2}$ distributions in Figs. 2, Here, the control-background sample was selected in the region $\theta^{2}=(0.1-0.2)\left[\right.$ degree $\left.^{2}\right]$. The background-subtracted signals are shown. Both 2004 and 2006 data have statistically insignificant excesses near $\theta^{2}=0$. The $\chi^{2} /$ DOFs (degree of freedom) for null assumptions are 18.6/15 and 13.2/15 for 2004 and 2006, respectively. These statistically insignificant excesses appear as the small excess count peaks around the centers of the plots in Fig. 1. Note that our PSF corresponds to $\theta^{2}<0.06$ degree $^{2}$. We, therefore, proceed assuming that there is no signal present. In order to show the consistency between 2004 and 2006, the vertical unit is unified to count rate.

In order to determine whether or not there is a gammaray excess around SN 1987A, we made the $F D$ distributions within the PSF. The control-background was made from the region $\theta^{2}=(0.1-0.2)$. The fitting results are shown in Fig. 3. The background level differs between the two years. This is due to sky conditions, mirror reflectivity, time-dependence of the blur spot size (which improves after maintenance periods), etc. These are considered to be the major systematics for estimating the observational gamma-ray flux in this work. For the light correction efficiency, we may have $10 \%$ uncertainty at maximum.

Again there is no statistically significant excess. We carried out a similar analysis, year by year, energy threshold by energy threshold. The obtained $2 \sigma$ upper limits (ULs) are listed in Table 1. Here we used a $E^{-2.1}$ spectrum for the gamma-ray simulation. The ULs range from $6-17 \%$ crab. The worse upper limits in the lower energy range originated from the statistically insignifi-
TABLE 1

The $2 \sigma$ UPPER Limits to THE INTEGRAL FLUXES From SN1987A AT TWO EPOCHS WITH THREE DIFFERENT ENERGY THRESHOLDS.

\begin{tabular}{cccc}
\hline \hline Year & $\begin{array}{c}\text { Excess Upper Limit } \\
\text { Events }\end{array}$ & $\begin{array}{c}\text { Energy Threshold } \\
\text { GeV }\end{array}$ & $\begin{array}{c}\text { Flux Upper Limit } \\
\mathrm{cm}^{2} \mathrm{~s}^{-1}\end{array}$ \\
\hline 2004 & 35 & 1200 & $1.7 \times 10^{-11}$ \\
2004 & 21 & 2870 & $5.7 \times 10^{-13}$ \\
2004 & 4.6 & 7180 & $1.0 \times 10^{-13}$ \\
2006 & 116 & 1080 & $2.7 \times 10^{-12}$ \\
2006 & 19 & 2550 & $2.5 \times 10^{-13}$ \\
2006 & 5.3 & 6350 & $6.1 \times 10^{-14}$ \\
\hline \hline
\end{tabular}

cant excess around the center of the field of view. At higher energies, we do not see any excess.

The four other high energy sources in the surrounding region, 30 Dor C, PSR B0540-69, LMC X-1, and N157B, can be analyzed in the same way. They are all within a one degree circle from the average pointing position (the sensitive region). The summary is listed in Table 2 These are two year averages. Note that, considering our

TABLE 2

The $2 \sigma$ UPPER LIMITS TO THE INTEGRAL FLUXES AT ENERGY GREATER THAN 1120 GEV FOR FIVE HIGH ENERGY OBJECTS. THE 2004 AND 2006 DATA WERE AVERAGED.

\begin{tabular}{ccc}
\hline \hline Target & $\begin{array}{c}\text { Excess Upper Limit } \\
\text { Events }\end{array}$ & $\begin{array}{c}\text { Flux Upper Limit } \\
\mathrm{cm}^{-2} \mathrm{~s}^{-1}\end{array}$ \\
\hline SN 1987A & 126 & $1.8 \times 10^{-12}$ \\
30 Dor C & 165 & $2.5 \times 10^{-12}$ \\
PSR B0540-69 & 66 & $1.1 \times 10^{-12}$ \\
LMC X-1 & 74 & $1.1 \times 10^{-12}$ \\
N157B & 167 & $2.4 \times 10^{-12}$ \\
\hline \hline
\end{tabular}

PSF of 0.24 degree, SN 1987A and 30 Dor C are highly confused, with other pairs being less confused. Also some of the control-background regions are confused. The acceptance is a slowly and smoothly decreasing function and is reduced to $65 \%$ at a distance of one degree from the center.

\section{DISCUSSION}

Our morphology indicates a negligibly small excess near the center of field of view both in the 2004 and 2006 data. This is statistically $<2 \sigma$ each year. We note that a similar feature can also be seen in the H.E.S.S. data shown in Figs. 2 and 3 of Rowell et al. (2005). A deep survey, i.e., longer observation, around this region is awaited. The region around LMC X-1 is consistent with zero in our data.

The upper limits of SN 1987A in Table 1 are plotted in Fig. 4. The results presented here (in blue and black) are compared with CANGAROO-II (red) (Enomoto et al. 2003) and H.E.S.S. (green) (Rowell et al. 2005) results. The upper limits in this work are improved by factors of up to 10 on the 2001 result. That is consistent with the longer observation time and better signal-to-noise ratio resulting from the three-fold coincidence. However, the theoretical prediction even 7300 days after the supernova (the dashed curve) (Berezhko \& Ksenofontov 2006) is still a factor of three below the results of the observations. Note that the predictions (Berezhko \& Ksenofontov 2006) were made in the frame of spherical symmetric approach. The existence of strongly asymmetric interstellar matter struc- 

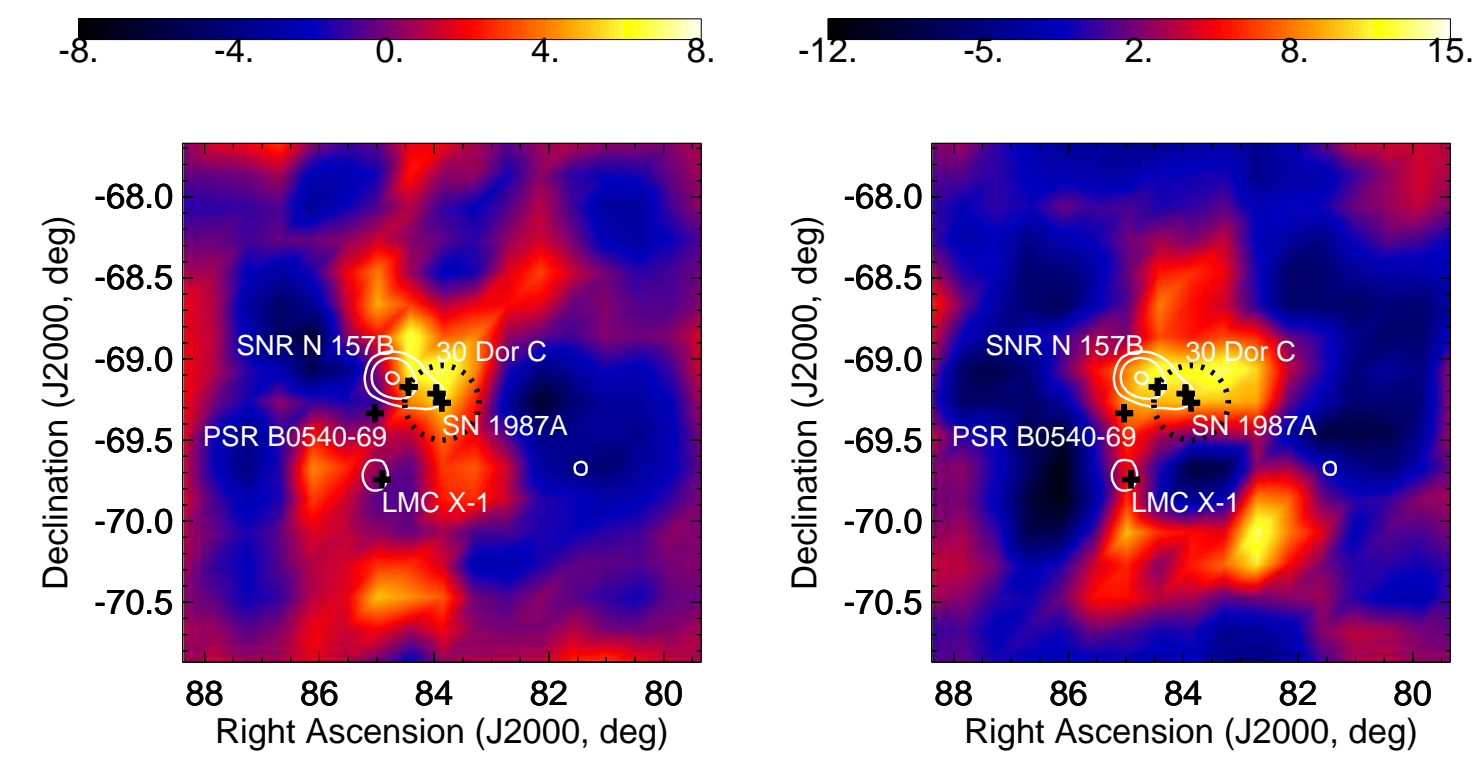

Fig. 1. - Excess count maps. The left panel is obtained from the 2004 data and the right from the 2006 data. The average pointing center is SN 1987A itself. The positions of four other high energy objects are also indicated by the black crosses with labels. The PSF is shown by the black dashed circle. The white contours are derived from $4.85 \mathrm{GHz}$ PMN survey radio data (Wright et al. 1994) obtained from Skyview (NASA) (NASA] 2007).

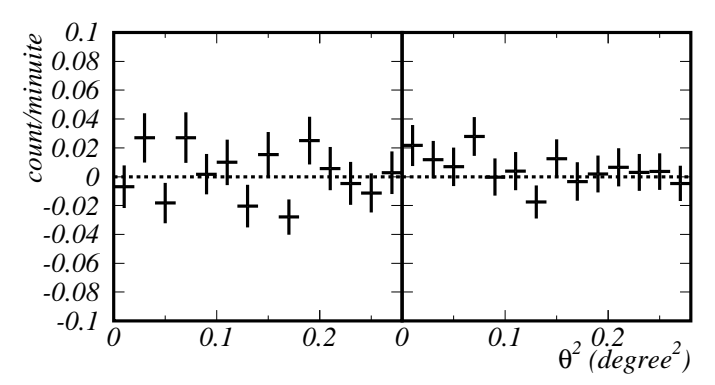

FIG. 2.- Distributions of $\theta^{2}$ [degree $\left.{ }^{2}\right]$. The left panel is obtained from the 2004 data and the right from the 2006 data. The vertical scale is normalized to counts/minute per bin $\left[0.2\right.$ degree $\left.^{2}\right]$. The background-subtracted signals (obtained by the fitting procedure described in the text) are plotted.

ture, which is a dense inner ring, makes their prediction of gamma-ray flux uncertain. According to their rough estimation, this uncertainty is not very large (about a factor of 2) due to the stronger supernova shock deceleration in the denser medium. The solid curve is their prediction for 8249 days after the supernova, still lower than the upper limits obtained in this work. In order to constrain this model, we need further improvements on the hardware and/or analysis methods. A deeper observation by H.E.S.S., such as T >30 h, around 2010 might give conclusive results. Also proposed future large-scale IACT arrays such as CTA (Hermann 2007) would be very promising.

The limit obtained for 30 Dor $\mathrm{C}$ in Table 2 is about $15 \%$ crab level at $\sim 1 \mathrm{TeV}$. Considering the distance of $50 \mathrm{kpc}$ to LMC, this upper limit corresponds to a huge cosmic-ray density comparing with that of the Crab neb-

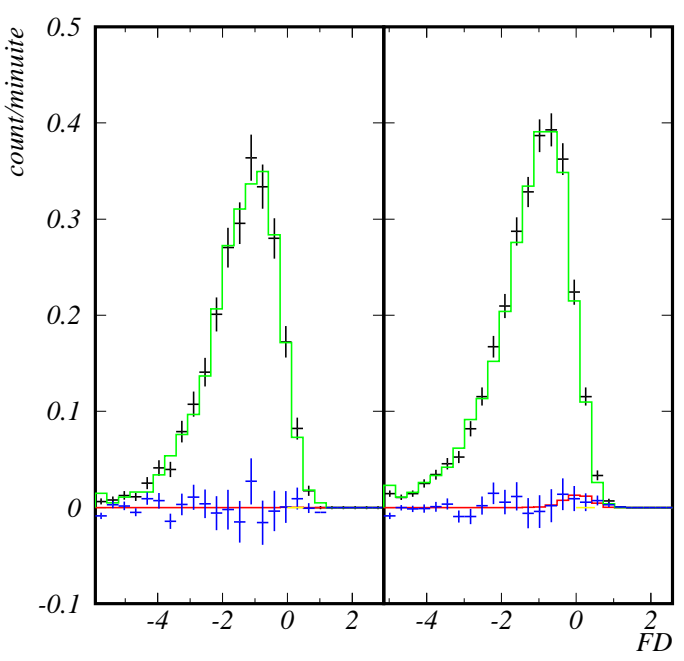

FIG. 3.- FD distributions. The left panel is obtained from the 2004 data and the right the 2006 data. The vertical scale is counts/minute per bin [arbitrary unit]. The black crosses are obtained from the on-source region (centered around SN 1987A within the PSF), the green histograms the best fit of the control backgrounds, the blue crosses the background-subtracted signals, and the red histograms the best fit signal distributions.

ula (distance of $2 \mathrm{kpc}$ ). Even if there is supernova complex there, the expected TeV gamma-ray flux is still below our sensitivity limit. Increasing the cosmic-ray target density such as either inter-stellar-matter or photon density within plausible ranges, the expected flux does not exceed this high upper limit. According to the standard estimation on the hadronic gamma-ray production such as Drury, Aharonian, \& Völk (1994); Naito \& Takahara 


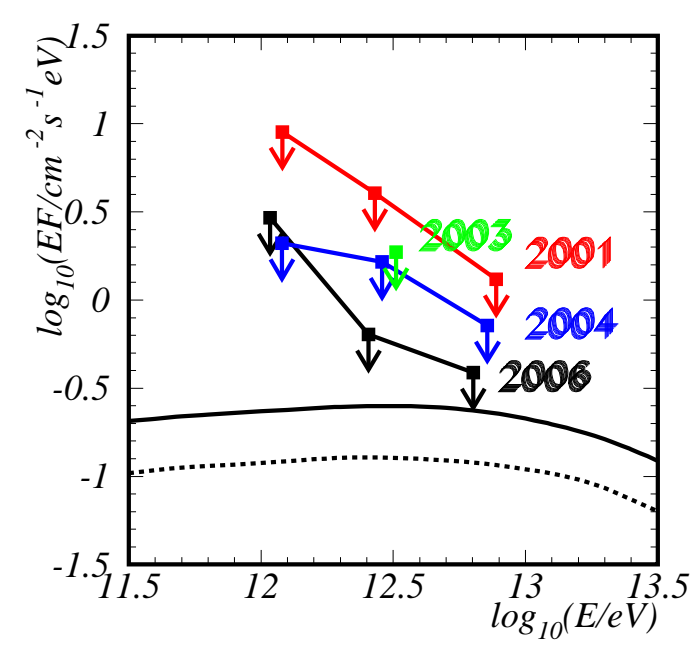

FIG. 4. - Spectral energy distribution. The blue points and line are obtained from the 2004 data and the black from the 2006 data. The red points are the CANGAROO-II upper limits from 2001 data (Enomoto et al. 2003). The green points are the H.E.S.S. data from 2003 (Rowell et al.|2005). The lines are obtained from Fig. 4 of Berezhko \& Ksenofontov (2006). The solid and dashed curves are the predictions of the gamma-ray flux 8249 and 7300 days after the supernova, respectively.

(1994) with an assumption of the target density of $1 \mathrm{p} / \mathrm{cm}^{-3}$, a total kinetic explosion energy of $7 \times 10^{51} \mathrm{ergs}$ gives a 1 milli-crab flux of gamma-rays at the solar system. Again future larger-scale projects are required to probe such flux levels.

For the other three high energy objects, we set upper limits far below those of previous observations. We, however, could not find any physically important constraints on their activity at high energies again due to the distance of $50 \mathrm{kpc}$.

\section{CONCLUSION}

We have observed SN 1987A at two epochs: MJD 53320-53323 (2004 Nov) and MJD 54081-54096 (2006 Dec), approximately 6500 and 7200 days after the supernova, respectively. The effective observation times are 10.5 and $20 \mathrm{~h}$, respectively. No statistically significant gamma-ray signals were obtained and we set $2 \sigma$ upper limits on the integral fluxes of $6-17 \%$ crab at $\sim 1 \mathrm{TeV}$. These are slightly lower than the previous CANGAROOII and H.E.S.S. upper limits. Although theory predicts a factor of $\geq 3$ lower flux 7300 days after supernova, it also predicts that the future flux level might exceed the sensitivity limit of the existing and future arrays. Continued monitoring of this object at $\mathrm{TeV}$ energies is still therefore meaningful in constraining theoretical models. It is also important to improve the sensitivity of the observation methods, as proposed by the CTA project (Hermann 2007).

In addition, we set $2 \sigma$ upper limits for four more high energy objects inside our field of view: 30 Dor C (super bubble), PSR B0540-69 (Crab-like pulsar), LMC X-1 (X-ray binary), and N157B (supernova remnant).

We thank L.T. Ksenofontov for discussion on the estimated gamma-ray flux from SN 1987A. This work was supported by a Grant-in-Aid for Scientific Research by the Japan Ministry of Education, Culture, Sports, Science and Technology, the Australian Research Council, JSPS Research Fellowships, and the Inter-University Research Program of the Institute for Cosmic Ray Research. We thank the Defense Support Center Woomera and BAE Systems.

\section{REFERENCES}

Bamba, A., Ueno, M., Nakajima, H., \& Koyama, K., 2004, ApJ, 602, 257

Berezhko, E.G., \& Ksenofontov, L.T., 2006, ApJ, 650, L59

Burrows, C. J., et al. 1995, ApJ, 452, 680

Daum, A., et al. 1997, Astropart. Phys., 8, 1

Drury, L.O'C., Aharonian, F.A., \& Völk, H.J., 1994, A\&A, 287, 959

Enomoto, R., et al. 2002a, Nature, 416, 823

Enomoto, R., et al. 2002b, Astropart. Phys., 16, 235

Enomoto, R., et al., 2003, ApJ, 591, L25

Enomoto, R., et al. 2006a, ApJ, 638, 397

Enomoto, R., et al. 2006b, ApJ, 652, 1268

Fisher, R. A. 1936, Annals of Eugenics, 7, 179

Habert, F., Geppert, U., Aschenbach, B., \& Hasinger, G., 2006, A\&A, 260,811.

Hermann, G., 2007, Cherenkov Telescope Array (CTA),

http://www.mpi-hd.mpg.de/htm/CTA/

Hillas, A. M. Proc. 19th Int. Cosmic Ray Conf. (La Jolla) 3, 445

Kabuki, S., et al. 2003, Nucl. Instrum. Meth., A500, 318

Kabuki, S., et al. 2007, ApJ, v667, in press (arXiv:0706.0367)

Kawachi, A., et al. 2001, Astropart. Phys., 14, 261

NASA, 2007, SkyView, http://skyview.gsfc.nasa.gov/

Morris, T., \& Podsiadlowski, P., 2007, Science, 315, 1103

Naito, T. \& Takahara, F., 1994, J. Phys. G., 20, 477
Nishijima, K., et al. 2005, Proc. 29th Int. Cosmic Ray Conf. (Pune), OG2.7, 101

Parizot, E., Marcowith, A., van der Swaluw, E., \& Tatischeff, V., 2004, A\&A, 424, 747

Park, S., Burrows, D.N., Garmire, G.P., McCray, R., Racusin, J.L., \& Zhekov, S.A., 2007, To appear in the proceedings of "Supernova 1987A: 20 Years after Supernovae and Gamma-Ray Bursters", held in Aspen CO. USA, Feb 19-23, arXiv:0704.0209

Rowell, G., Hinton, J., Benbow, W., \& H.E.S.S. Collaboration, 2005, in AIP Conf. Proc. 745, High Energy Gamma-Ray Astronomy, ed. F.A. Aharonian, H.J. Völk, \& D. Horns (Melville: AIP), 299

Staveley-Smith, L., Ball, L., Gaensler, B., Kestreven, M., Manchester, D., \& Tzioumis, T., 2007, ATNF News, Issue No. 61, ISSN 1323-6326, http://www.atnf.csiro.au/news/newsletter/feb07/

Ueno, M., Bamba, A., \& Koyama, K., 2003, in "The Universe Viewed in Gamma-Rays", Proc. Univ. of Tokyo Symposium, ed. R. Enomoto, M. Mori, \& S. Yanagita (Universal Academy Press: Tokyo), 311

Wright, A. E., Griffith, M. R., Burke, B. F., \& Ekers, R. D. 1994, ApJS, 91, 111 